\title{
Entre hommes et femmes médecins, des intérêts clivés?
}

\author{
Jürg Schlup \\ Dr méd., président de la FMH
}

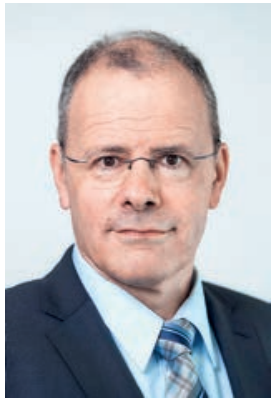

Certains raisonnements semblent évidents. Jusqu'à peu, par exemple, la distribution inégale entre hommes et femmes en médecine selon les disciplines me paraissait refléter avant tout des aspirations différentes. Sinon, pourquoi nos consœurs seraient-elles représentées de manière proportionnelle, voire surreprésentées dans certaines disciplines comme la médecine de famille ou la pédiatrie et, en revanche, sous-représentées dans les disciplines chirurgicales? Cependant, à y regarder de plus près, ce phénomène est plus complexe. Le choix d'une spécialité, ou d'autres décisions de carrière, est tributaire de nombreux facteurs, comme le montre l'article à la page 246: d'abord l'intérêt pour une discipline, mais ce n'est pas la seule raison. Beaucoup optent pour plusieurs disciplines et, finalement, les conditions cadres vont aussi guider nos choix.

Au moment de choisir une spécialisation, l'intérêt pour la discipline est déterminant, mais aussi les conditions cadres.

Deux aspects peuvent peser lourdement. D'abord, quelles restrictions de ma vie privée imposerait la spécialisation retenue? Quels sacrifices serais-je prêt à consentir? Mais aussi quelles possibilités de carrière m'offrirait cette discipline? Est-ce que je serai soutenu ou plutôt freiné? Pourrai-je compter sur une saine concurrence pour les postes attractifs? Leur pondération variera si ces questions sont posées à des femmes ou des hommes.

Le premier aspect est lié à l'organisation du temps de travail. Ceux qui opèrent, puis accompagnent leurs patients pendant de longues heures de jour et de nuit ont inévitablement moins de temps pour leur vie privée. Et si ces périodes sont peu prévisibles, il sera encore plus difficile d'assumer des responsabilités familiales. La jeune génération de médecins ferait bien de poser la question de la nécessité de ces conditions de travail, mais aussi à quelle tradition et à quelle culture professionnelle elles répondent: faut-il vraiment des semaines de travail de plus de 50 heures pour savoir bien opérer? Pourquoi les médecins-assistants consacrent-ils autant de temps aux tâches administra- tives? Ces conditions cadres ont certainement déjà incité de jeunes talents à se détourner vers d'autres spécialités.

La mise en place de conditions de travail plus favorables gagne en importance parce que, depuis 2004 , une majorité croissante de diplômes de médecins ont été décernés à des femmes. Mais les hommes sont également mis au défi aujourd'hui, car le modèle du salaire unique, avec des femmes au foyer rendant possible ces très longs horaires, est désormais dépassé. Avec la vie professionnelle des femmes, l'équilibre entre le travail et la famille devient aussi un problème masculin, sans compter que, de manière générale, l’importance accordée à la vie privée augmente.

Le deuxième aspect concerne la promotion et les chances de réussite. Les jeunes femmes sont trop souvent désavantagées, surtout dans les disciplines chirurgicales, par la crainte d'une pause pour se consacrer à la famille et d'une réduction même momentanée de leur engagement. Si l'on ajoute à cela le peu de modèles féminins, il est probable que certaines chirurgiennes talentueuses n'iront pas plus loin dans une discipline qui pourtant les aurait intéressées.

Mais cette situation est en train d'évoluer. En 2017, 49\% des titres de spécialiste en chirurgie ont été attribués à des femmes, et nos consœurs investissent progressivement des domaines traditionnellement masculins, comme la chirurgie orthopédique: pour une proportion de femmes de 9,5\% dans cette discipline, $31 \%$ des titres de spécialiste ont été attribués à des femmes en 2017.

\section{Pourrai-je compter sur une saine concurrence pour les postes attractifs?}

Cela vaut donc la peine de porter un regard plus précis et de réviser nos préjugés. Et c'est primordial lorsque nous recherchons de nouvelles solutions pour la formation postgraduée et le temps de travail, afin de susciter l'envie et le talent des jeunes générations de médecins. Au moment de choisir une discipline, aucun obstacle inutile ne devrait barrer la route vers ce qui nous intéresse. 